Case Series

\title{
Acute limb ischemia in COVID-19 patients and its rehabilitation: a case series from a tertiary care hospital in Central India
}

\author{
Rohit Harchandani*, Manoj Kela, Abhishek Bawa, Ashish Rathod, Sheikh Wajid, Apoorv \\ Gupta, Rohan Chaphekar, Vinod Bhandari
}

Department of General Surgery, Sri Aurobindo Institute of Medical Science, Indore, Madhya Pradesh, India

Received: 15 July 2021

Revised: 13 August 2021

Accepted: 26 October 2021

\section{*Correspondence:}

Dr. Rohit Harchandani,

E-mail: research.drrharchandani@gmail.com

Copyright: (C) the author(s), publisher and licensee Medip Academy. This is an open-access article distributed under the terms of the Creative Commons Attribution Non-Commercial License, which permits unrestricted non-commercial use, distribution, and reproduction in any medium, provided the original work is properly cited.

\begin{abstract}
Severe acute respiratory syndrome coronavirus 2 (SARS-CoV-2) is a highly transmissible and infectious virus. It belongs to the family coronavirus and it is a single strand positive sense virus. There are many theories which relate to the origin and transmission of this virus and hence the virus has been tagged with many names according to various taxonomical guidelines. The conventional clinical spectrum of this infection includes cough, fever, dyspnea, anorexia and gastrointestinal symptoms. It has been seen during the course of this disease that there are also some properties relating this virus to the hypercoagulability in the disease spectrum. The cases involving arterial and venous thromboembolism are not rare. This case series is a collection on three such cases from the red zone (zone separated for COVID-19 infected patients) of a tertiary care hospital in central India which showcase acute limb ischemia in patients infected with SARS-CoV-2.
\end{abstract}

Keywords: COVID-19, SARS-CoV-2, Acute limb ischemia, Thromboembolism, Hypercoagulability

\section{INTRODUCTION}

Since December 2019, when world saw its first case of this mysterious pneumonia, many stories have been unfolded regarding this novel virus. ${ }^{1}$

The virus that was responsible for this fatal disease was identified to as SARS COV 2 or COVID $19 .{ }^{1}$ Starting its journey from its first case noticed in Wuhan, China till today when the world is suffering manifolds from this global pandemic, there are many chapters in the story of this virus is continuously being studied. ${ }^{2}$ The virus is observed giving rise to a myriad of coagulation abnormalities along with the constitutional respiratory tract infections that we are aware of. ${ }^{3}$

Mechanisms starting from derangements in D dimer and fibrinogen values, lead to repercussions as harmful as large arterial and venous thrombus formation. ${ }^{4}$ This case series is a collection of three such cases where this virus has been life challenging.

\section{CASE SERIES}

\section{Case 1}

A 52 years old male, was admitted to our hospital with complains of Breathlessness and fever. During evaluation for his pyrexia and dyspnea, he was investigated for SARS-CoV-2 RNA by RT-PCR method and was tested positive for the same.

The patient gave no history of contact with a positive case or travel to a high-risk zone. He had no comorbidities with no history of addictions such as consumption of alcohol or tobacco in any form. On the next day of admission, the patient complained of pain in right lower limb. On examination, it was noted that the local temperature of the right lower limb below the knee was lesser when compared 
to the opposite limb and the same limb over the knee level. It was also noted that gangrenous changes were present over the plantar aspect of the right foot.

The peripheral pulsations in the dorsalis pedis anterior and posterior tibial, popliteal and femoral arteries were not present in the right lower limb. Intravenous heparin therapy was given.

The patient was planned to undergo a right lower limb CT angiogram after all the necessary investigations which revealed complete absence of flow enhancement in the right deep femoral, superficial femoral, popliteal, anterior and posterior tibial arteries. Right common and external iliac arteries revealed complete absence of flow enhancement. Right internal iliac artery showed normal flow enhancement.

It was noted that distal reformation of right common femoral artery was present, through collaterals, which revealed normal flow enhancement for short course. Few muscular branches were also noted in the right thigh region which showed normal flow enhancement. Right common and external iliac artery revealed complete absence of flow enhancement.

Right internal iliac artery showed normal flow enhancement. During the entire study, left common, external, internal iliac arteries, left common femoral artery superficial femoral artery and deep femoral artery showed normal flow enhancement. The patient was advised for right lower limb above knee amputation.

A written and informed consent was obtained. Intra operatively gangrenous muscles and tissued were seen till the level of right mid-thigh. Post operatively patient was stable. Regular sterile dressings were done and a healthy suture line was maintained. No blackening of the suture line was observed.

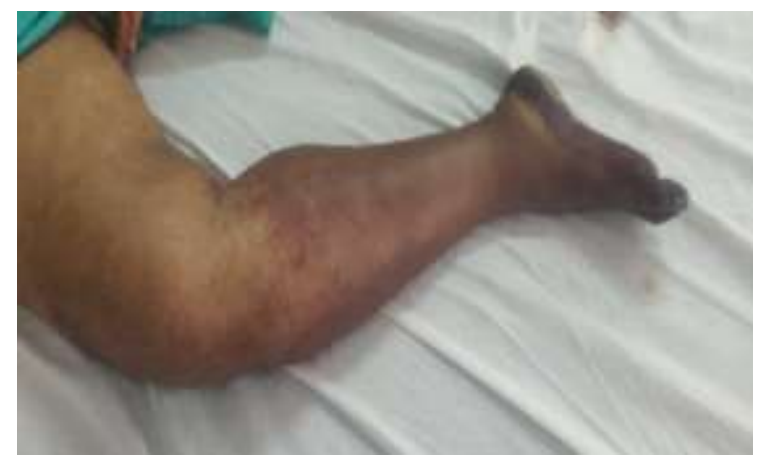

Figure 1: Image showing right lower limb of a 52 years old male suffering from COVID-19 infection. The limb on examination shows signs of acute limb ischemia which include dusky changes over the skin with blackening of skin over the sole. It is to be noted that the limb shows no signs of chronic ischemia like loss of hair and other associated skin changes.

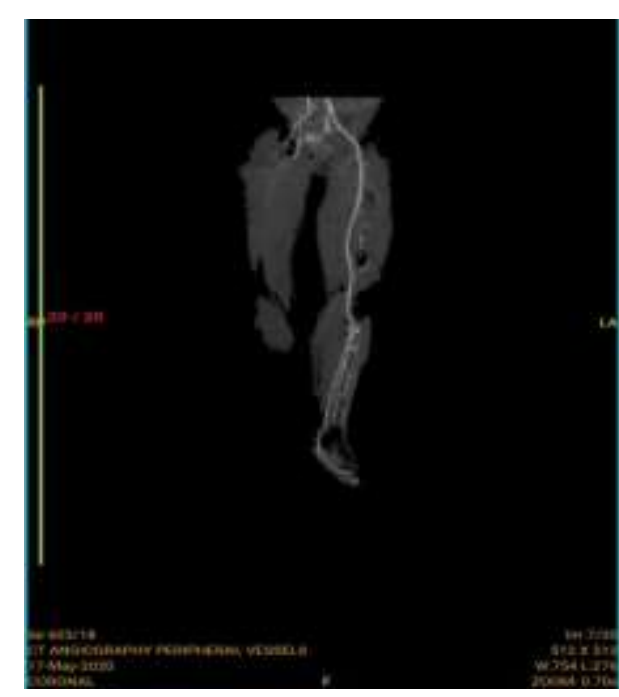

Figure 2: Computed tomography angiogram scan showing complete absence of flow below the level of common and external iliac arteries.

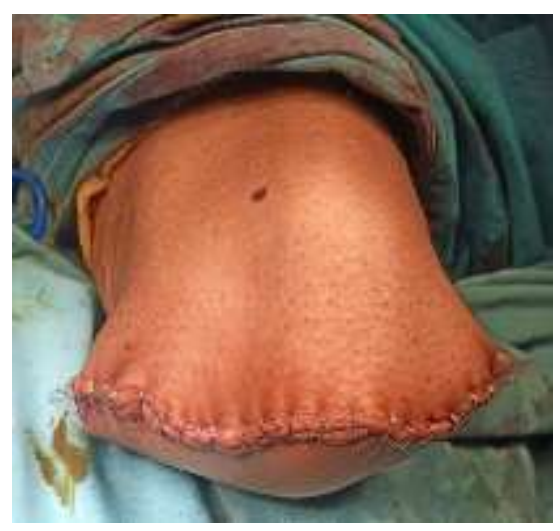

Figure 3: Right lower limb above amputation stump with healthy suture line.

\section{Case 2}

A 39 years old male was admitted to the hospital with complains of fever and on evaluation was tested positive for SARS-CoV-2 RNA by RT-PCR method. On examination it was noted that the patient developed blackening of the right lower limb $10 \mathrm{~cm}$ below the tibial tuberosity. However, the patient did not complain of any pain in the right lower limb. On palpation, local temperature was found to be decreased in the left lower limb.

There were no signs of chronic limb ischemia. Peripheral pulsations were normal in the left lower limb and absent in femora, popliteal, anterior and posterior tibial and dorsalis pedis arteries of right lower limb.

On CT angiography severe thrombo occlusion was seen in the right infra renal aorta involving approximately $5.2 \mathrm{~cm}$. Severe thrombo occlusion was present in the common and external iliac arteries bilaterally. In the right lower limb, 
partial reformation of right common femoral artery was seen with approximately $2 \mathrm{~cm}$ length of the proximal segment. Followed by severe thrombo embolism seen in right superficial femoral, right popliteal and anterior and posterior tibial arteries.

During the entire study, left common, external, internal iliac arteries, left common femoral artery superficial femoral artery and deep femoral artery showed normal flow enhancement. There was no evidence of deep venous thrombosis in the doppler study bilaterally.

Meanwhile, heparin infusion therapy was started. The patient was advised for right above knee amputation and was counselled regarding the need for bilateral lower limb amputations. The patient underwent above knee amputation of the right upper limb. Post-operative period was uneventful. Healthy suture line was maintained. No blackening of the suture line was observed.

\section{Case 3}

A 43 years old male patient, came with symptoms suggestive of COVID-19 infection. He was tested and was proven positive for COVID-19 by RT-PCR method. He developed sudden weakness of left lower limb followed by pain in left lower limb which was severe in intensity. On examination it was observed that tenderness was not unobvious.

On palpation all the peripheral pulses below the level of femoral artery in the affected limb were non-palpable. Loss of normal temperature was noted in the affected limb up till the thigh. On evaluation by CT angiography, it revealed that proximal part of the left common iliac artery showed normal enhancement.

The remaining left common iliac artery, i.e.; exempting the proximal $2.6 \mathrm{~cm}$, revealed complete absence of flow enhancement. The left internal iliac artery also showed complete loss of flow enhancement/occlusion. Distal reformation/normal flow was seen in left common femoral, deep femoral and superficial femoral arteries.

Complete absence of flow enhancement was seen in left popliteal, anterior tibial and posterior tibial artery. Spars opacification was seen in terminal anterior and posterior tibial and dorsalis pedis artery. Patient was started on heparin infusion therapy with regular monitoring and titration on the basis of APTT, which was monitored every sixth hourly.

He was planned for embolectomy and above knee amputation of left lower limb considering all the possible risks. Embolectomy of left femoral artery with fasciotomy in the left calf region was done. But the limb showed signs of irreversible ischemia and as the last resort, left lower limb above knee amputation was done under spinal anesthesia. Post-operative period was uneventful. No blackening of suture line was observed.

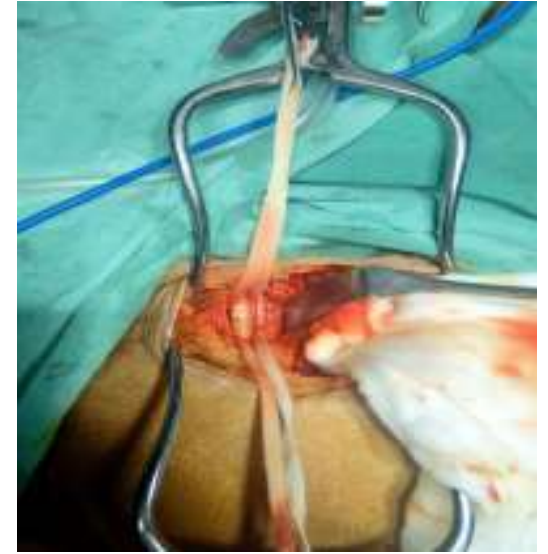

Figure 4: Intra-operative image of left femoral arteriotomy.

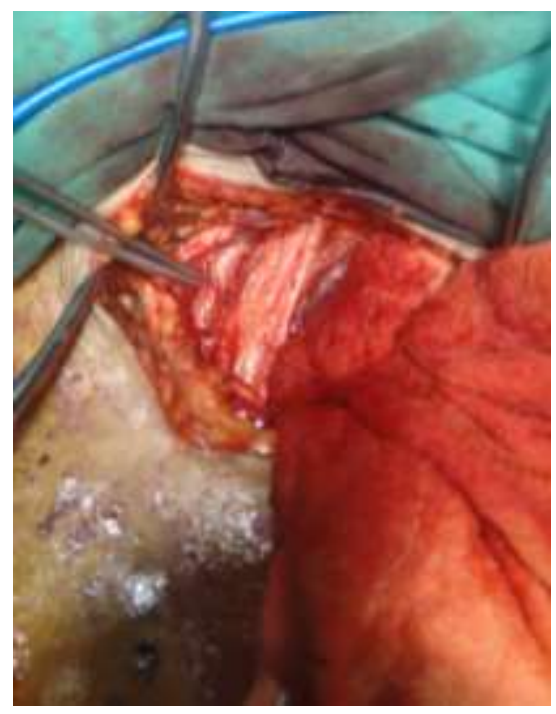

Figure 5: Image post left femoral arteriotomy.

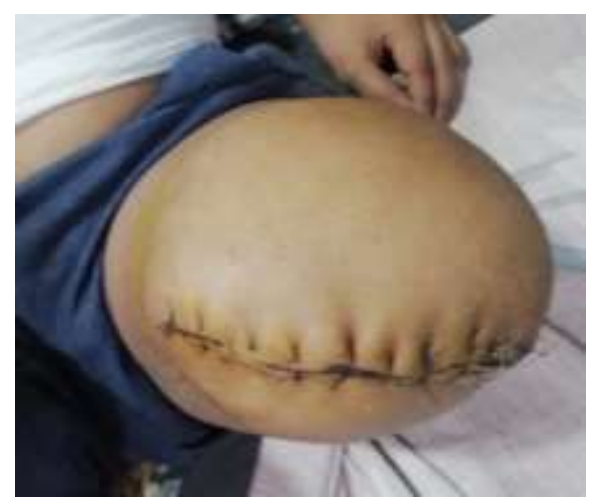

Figure 6: Left lower limb above knee amputation stump with healthy suture line showing no signs of necrosis over the suture line.

\section{DISCUSSION}

SARS-CoV-2 is a continuously evolving virus. One of the key mechanisms of this virus involves the triggering of inflammatory cascade which further damages the 
endothelium leading to the start of virchows triad (stasis of blood flow, endothelial injury and hypercoagulability). ${ }^{5}$ When the inflammatory process damages the linings of micro and macro vasculature, it leads to disturbance of anti-thrombotic and anti-inflammatory mechanisms resulting in unchecked activation of thrombotic cascade. ${ }^{6}$ While, the virus is known to cause a family of symptoms that occur commonly including fever, headache, anosmia, shortness of breath, anorexia, the symptoms occurring from a myriad of coagulation disorders are not uncommon. ${ }^{5}$ The ACE-2 receptors, which are normally present in the human body and are expressed in various degrees in alveolar epithelial cells, large and small arterial endothelial cells, small intestinal epithelial cells, immune tissues, and various other types of cells serve as the entry point for SARS-CoV-2 proteins. ${ }^{7}$ These receptors, when present on the vascular endothelial cells, supports the SARS-CoV-2 to activate coagulation cascade after causing endothelial injury. ${ }^{8}$

The insult, leads to excessive cytokine release leading to a cytokine storm and further activating the coagulation factors. It also inhibits fibrinolysis, leading to DIC (Disseminated intravascular coagulation) like extensive thrombosis. IL-6 plays a pivotal role in SARS-CoV-2 induced inflammatory mechanisms. IL-6, through various mechanisms stimulates the liver to generate fibrinogen and thrombopoetin. It is also known to upregulate the expression of VEGF (vascular endothelial growth factor) which further stimulates the monocytes to express increased amounts of tissue factors and thereby activating the extrinsic pathway of coagulation. ${ }^{8}$ Elevated D-dimer values with coagulation abnormalities should be considered as indicators for higher mortality predisposing patients to various ischemic and thrombotic events. ${ }^{8}$ The progression of sepsis induced prothrombotic state secondary to SARS-CoV-2 can be described by elevated Prothrombin time (PT), Partial thromboplastin time (PTT), fibrinogen, Fibrin degradation products (FDP), D-dimer and IL-6. ${ }^{8}$ The mainstay role of IL-6 in the inflammatory mechanism justifies the use of low molecular weight heparin, which acts as an inhibitor of IL-6, in the treatment of this myriad of symptoms. ${ }^{8}$

It is still to be noted that amongst all the three cases that are being discussed, despite the use of low molecular weight heparin and later unfractionated heparin, the patient's gangrene continued to worsen, ultimately requiring vascular interventions. There are studies that support the anticoagulation therapy as a prophylaxis for the venous thromboembolism. But it still remains unclear, that arterial thrombosis would be benefitted by a prophylactic or full anticoagulation therapy or not. The current scenario, which was common amongst all three cases suggests to divided conclusions on the basis of viability of the tissue. The patients with viable tissue, undergo a vascular intervention for arterial thrombolysis and thrombectomy. Limbs with irreversible ischemia and non-viable tissue lead the surgeon with amputation as the last resort, as in our cases.

\section{CONCLUSION}

The study summarises as the need of vigilance with the atypical presentations of the active COVID-19 and those who have recovered from the active COVID-19 infection. The need of anticoagulation therapy justifies as the mainstay of treatment in both, the primary pulmonary and atypical complications while acting as IL-6 inhibitor. In such cases, where one limb has undergone irreversible loss, the other organs can be prevented to suffer the damage.

\section{Funding: No funding sources \\ Conflict of interest: None declared \\ Ethical approval: Not required}

\section{REFERENCES}

1. Yüce M, Filiztekin E, Özkaya KG. COVID-19 diagnosis -A review of current methods. Biosens Bioelectron. 2021;172:112752.

2. Bellosta R, Luzzani L, Natalini G, Pegorer MA, Attisani L, Cossu LG, et al. Acute limb ischemia in patients with COVID-19 pneumonia. J Vasc Surg. 2020;72(6):1864-72.

3. Sánchez JB, Cuipal AJD, Ramos IR, Luna CZ, Cubas WS, Coaguila CA, et al. Acute Limb Ischemia in a Peruvian Cohort Infected by COVID-19. Ann Vasc Surg. 2021;72:196-204.

4. Avila J, Long B, Holladay D, Gottlieb M. Thrombotic complications of COVID-19. Am J Emerg Med. 2021;39:213-8.

5. Etkin Y, Conway AM, Silpe J, Qato K, Carroccio A, Manvar SP, et al. Acute Arterial Thromboembolism in Patients with COVID-19 in the New York City Area. Ann Vasc Surg. 2021;70:290-4.

6. Kaur P, Qaqa F, Ramahi A, Shamoon Y, Singhal M, Shamoon F, Maroules M, et al. Acute upper limb ischemia in a patient with COVID-19. Hematol Oncol Stem Cell Ther. 2020;1658.

7. Avila J, Long B, Holladay D, Gottlieb M. Thrombotic complications of COVID-19. Am J Emerg Med. 2021;39:213-8.

8. Anwar S, Acharya S, Shabih S, Khabut A. Acute Limb Ischemia in COVID-19 Disease: A Mysterious Coagulopathy. Cureus. 2020;12(7):9167.

9. Mietto C, Salice V, Ferraris M, Zuccon G, Valdambrini F, Piazzalunga G, et al. Acute Lower Limb Ischemia as Clinical Presentation of COVID19 Infection. Ann Vasc Surg. 2020;69:80-4.

Cite this article as: Harchandani R, Kela M, Bawa A, Rathod A, Wajid S, Gupta A, et al. Acute limb ischemia in COVID-19 patients and its rehabilitation: a case series from a tertiary care hospital in Central India. Int J Res Med Sci 2021;9:3676-9. 